\title{
Dissociation between airway responsiveness to methacholine and responsiveness to antigen
}

\author{
A. Kamachi*, Y. Nasuhara*, M. Nishimura*, T. Takahashi*, Y. Homma", Y. Ohtsuka\#, \\ M. Munakata\#
}

\begin{abstract}
Dissociation between airway responsiveness to methacholine and responsiveness to antigen. A. Kamachi, Y. Nasuhara, M. Nishimura, T. Takahashi, Y. Homma, Y. Ohtsuka, M. Munakata. (C) ERS Journals Ltd 2002.

ABSTRACT: Repeated aerosolized antigen challenges to brown Norway (BN) rats generate nonspecific airway hyperresponsiveness (AHR). On the other hand, some studies have demonstrated that repeated antigen challenge could attenuate antigenspecific AHR in BN rats. The authors questioned whether such dissociation in airway responses actually occurs when assessed in a single study in the same animals.

The authors simultaneously measured AHR to methacholine and antigen-specific AHR in rats that were repeatedly exposed to aerosolized ovalbumin (OA) for 1 or 3 months after sensitization. Four days after the last challenge, airway responses to methacholine and OA, morphometry of the airways, the cell profile in bronchoalveolar lavage fluid, and cytokine messenger ribonucleic acid (mRNA) expression in the lungs were evaluated.

The two types of AHR were modulated in opposite directions by repeated antigen challenges. The AHR to methacholine was significantly increased in the rats receiving antigen challenges compared with the control rats receiving saline challenges after sensitization; whereas, the antigen-specific AHR was significantly decreased. The number of alveolar macrophages in lavaged fluid and the expression of transforming growth factor- $\beta_{1}$ mRNA in lung tissue was significantly different between the antigenchallenged rats and the control rats.

In conclusion, dissociation between nonspecific airway hyperresponsiveness and antigen-specific airway hyperresponsiveness in brown Norway rats after repeated antigen challenges was demonstrated. Sustained airway inflammation with macrophages and/or upregulation of transforming growth factor- $\beta_{1}$ messenger ribonucleic acid in the lung tissue may be responsible for this dissociation.
\end{abstract}

Eur Respir J 2002; 19: 76-83.

\begin{abstract}
*First Dept of Medicine, Hokkaido University School of Medicine, Sapporo, Japan. ${ }^{\#}$ The Dept of Pulmonary Medicine, School of Medicine, Fukushima Medical University, Fukushima, Japan. The Medical Administration Center, Hokkaido University, Sapporo, Japan.
\end{abstract}

Correspondence: A. Kamachi

First Dept of Medicine

Hokkaido University School of Medicine N-15, W-7kita-ku,

Sapporo, 060-8638

Japan

Fax: 81117067899

Keywords: Airway hyperresponsive-

ness

macrophage

repeated antigen challenge

transforming growth factor- $\beta$

Received: January 292001

Accepted after revision October 32001
Brown Norway $(\mathrm{BN})$ rats have been used as a model of atopic asthma [1-3]. Sensitization and a subsequent single antigen challenge to these rats induce a number of immunological, physiological and pathological features similar to those observed in human allergic asthma. These include production of an antigen-specific immunoglobulin-E ( $\mathrm{IgE}$ ) antibody [1], upregulation of $\mathrm{T}$-helper 2 cytokines such as interleukin (IL)-4 and/or IL-5 [2], early and late phase airway reactions to the inhaled antigen [1], eosinophilic airway inflammation, and nonspecific airway hyperresponsiveness (AHR) to methacholine [3]. In the same strain of rats, multiple antigen challenges are reported by some investigators to develop chronic abnormalities resembling those in asthma, such as airway remodelling $[4,5]$. In addition, it has been demonstrated that nonspecific AHR occurs after multiple antigen challenges in some reports [4-8]. In contrast, there have been a number of studies, which observed the generation of $\mathrm{IgE}$ isotype-specific tolerance $[9,10]$ after multiple antigen challenges and also the attenuation of antigen-specific AHR.

Thus, the present authors were interested in the possible dissociation of nonspecific AHR from antigen-specific AHR in repeatedly antigen-challenged $\mathrm{BN}$ rats. The authors then attempted to examine the mechanism by which nonspecific AHR was changed in such desensitized animals. To achieve this goal, morphological changes of the airways, inflammatory cells in bronchoalveolar lavage fluid (BALF), and cytokine messenger ribonucleic acid (mRNA) expression in the lungs, which are considered to be the factors associated to the development of nonspecific AHR, were examined. It was anticipated that this study might provide insights into the reason why nonspecific AHR could remain increased under the condition of desensitization.

\section{Materials and methods}

\section{Immunization and airway sensitization}

All experimental protocols and procedures were approved by the Ethical Committee on Animal 
Research, Hokkaido University School of Medicine, Sapporo, Japan. Specific pathogen-free 6-week-old male BN rats (weight range 160-210 g) were purchased from Japan Charles River Co. (Yokohama, Japan). The rats were kept in a conventional colony in a Hokkaido University animal care facility. They were actively sensitized for ovalbumin (OA) by subcutaneous injection with $1 \mathrm{mg}$ of $\mathrm{OA}$ dissolved in gel including $200 \mathrm{mg}$ of aluminium hydroxide. An adjuvant $(1 \mathrm{~mL})$ consisting of $1 \times 10^{9}$ heat-killed Bordetella pertussis organisms was injected intraperitoneally at the same time. Two weeks after the sensitization, the rats inhaled $2 \%$ weight/volume OA for $15 \mathrm{~min}$ in an exposure chamber $(35 \times 25 \times 20 \mathrm{~cm})$ to sensitize the airway. For inhalation challenge, OA was dissolved in saline and aerosolized with an ultrasonic nebulizer (NE-U11B, Omuron Co., Tokyo, Japan), which delivered the aerosol at $1 \mathrm{~mL} \cdot \mathrm{min}^{-1}$. For repeated antigen exposure, $5 \% \mathrm{OA}$ was challenged for $10 \mathrm{~min}$.

\section{Experimental protocol}

Rats receiving a single OA challenge alone (single$\mathrm{OA}, \mathrm{n}=5$ ) were examined 4 days after the challenge to confirm increased airway responses to methacholine (Mch) and OA. For comparison, sham-sensitized and saline-challenged rats (single-sham, $n=4$ ) were also examined. To examine the effects of repeated antigen challenges, the other single-OA rats were divided into two groups, and they received further challenges with either OA or saline. One group of rats received repeated OA challenges every 2 days for 4 weeks (1M$\mathrm{OA}, \mathrm{n}=8)$ or 12 weeks (3M-OA, $\mathrm{n}=8$ ). The other group received saline instead of $\mathrm{OA}$ in an identical way (1Mcont, $\mathrm{n}=8 ; 3 \mathrm{M}$-cont, $\mathrm{n}=8$ ).

\section{Measurement of bronchial responsiveness to methacholine and ovalbumin}

Four days after the final challenge, the rats were anesthetized with an intraperitoneal injection of pentobarbital sodium $\left(50 \mathrm{mg} \cdot \mathrm{kg}^{-1}\right)$. Intratracheal intubation was then performed with a metallic tube ( $2 \mathrm{~mm}$ internal diameter). The rats were mechanically ventilated (Rodent Ventilator Model 683, Harvard Apparatus, Holliston, MA, USA) with a tidal volume of $6 \mathrm{~mL} \cdot \mathrm{kg}^{-1}$ and frequency of 100 breaths $\cdot \mathrm{min}^{-1}$. A pressure transducer (TP-602T, Nihon Kohden Co., Japan) was connected to a side port of the metallic tube, and airway opening pressure $(P$ ao $)$ was continuously measured. An aerosol of Mch or OA was produced using an ultrasonic nebulizer with an output of $0.2 \mathrm{~mL} \cdot \mathrm{min}^{-1}$ and administered through a reservoir box connected to the ventilator system. After measurement of baseline $P$ ao, an aerosol of saline followed by Mch was administered for $1 \mathrm{~min}$ in progressively doubled concentrations from $0.0625 \mathrm{mg} \cdot \mathrm{mL}^{-1}$. The airway response to Mch was assessed by the Mch concentration needed to induce a $50 \%$ increase from baseline $P$ ao $(\mathrm{PC} 150 \mathrm{Mch})$.

After the Mch inhalation test, the rats were forced to take deep breaths every $2 \mathrm{~min}$ for $>15 \mathrm{~min}$ until
$P$ ao returned to near the baseline level. Five per cent $\mathrm{OA}$ was then inhaled for $10 \mathrm{~min}$. The airway response to $\mathrm{OA}$ was expressed as the per cent increase of $P$ ao from before to after OA inhalation.

\section{Morphometric dimensions}

Immediately after the measurement of airway responsiveness, the rats were exsanguiated from the aorta, and the left lung was fixed with an intrabronchial infusion of $10 \%$ neutral formalin at a constant pressure of $25 \mathrm{cmH}_{2} \mathrm{O}$ for a period of $48 \mathrm{~h}$. Paraffinembedded midsagittal sections $(5 \mu \mathrm{m}$ thick) were then prepared and stained with haematoxylin and eosin in order to evaluate the density of eosinophils in the airways. Airway smooth muscle was stained with a monoclonal anti- $\alpha$ smooth muscle actin antibody (clone 1A4; DAKO Danmark A/S, Glostrup, Denmark) by the avidin-biotin alkaline phosphatase complex method (Vectastain ABC-AP kit; Vector Laboratories, Burlingame, CA, USA). Vector red (Vector Laboratories), which is highly fluorescent, was used as the substrate for alkaline phosphatase to differentiate airway smooth muscle from the surrounding connective tissue.

An image analyser (XL-10, Olympus Co., Tokyo, Japan) was used in combination with light/fluorescent microscopy. Only airways with a short-to-long diameter ratio of $\leqslant 0.5$ were analysed. Approximately 10 airways were measured for each rat. The internal and external perimeter (equal to the length of the basement membrane) of the epithelium, and the external perimeter of the airway wall were traced. The area of airway smooth muscle was measured after changing the microscopic view from light to fluorescence. Airways were divided into three groups by the length of the basement membrane $(\mathrm{BM})(\mathrm{S}: 0.5-1 \mathrm{~mm}$; $\mathrm{M}$ : 1-2 mm; L: $\geqslant 2 \mathrm{~mm}$ ). All areas were divided by the $\mathrm{BM}$ length ${ }^{2}$ to standardize the variation of airway size. Areas of airway smooth muscle, airway wall and epithelium were compared in the same-sized airways between the groups. Morphometry was evaluated by an independent researcher, who was unaware of the source of specimens at the time of evaluation.

\section{Bronchoalveolar lavage and cell counting}

Another set of six groups (single-sham, single-OA, 1M-cont, 1M-OA, 3M-cont, 3M-OA) was prepared to examine the profile of inflammatory cells in BALF and cytokine mRNA expression in the lungs.

Four days after the final aerosol challenge, the lungs were lavaged four times with a total volume of $20 \mathrm{~mL}$ of sterile saline, while a 16-gauge catheter was placed into the trachea. After the lavage, the right lungs were frozen for later studies of cytokine mRNA expression. The lavage fluid was centrifuged $\left(5 \mathrm{~min}, 500 \times g\right.$ at $\left.4{ }^{\circ} \mathrm{C}\right)$, and the cells were resuspended in $1 \mathrm{~mL}$ of Hanks' balanced solution (Cosmo Bio Co. Ltd, Tokyo, Japan). Cells were counted and processed for differential cell analysis using Giemsa staining by counting 300 cells. Flow cytometry was performed to count the 
number of CD4 and CD8 lymphocytes. The $5 \times 10^{5}$ cells in lavage fluid were stained with a fluorescein isothiocyanate-conjugated mouse antirat CD4 (OX-38) or CD8 (OX-8) monoclonal antibody (Pharmingen, San Diego, CA, USA) for $30 \mathrm{~min}$ at $4^{\circ} \mathrm{C}$. After washing with phosphate-buffered saline, flow cytometry was performed by fluorescence-activated cells sorting (FACS) using a FACScan analyser (Becton Dickinson FACS Division, Sunnyvale, CA, USA).

Real-time quantitative reverse transcriptase polymerase chain reaction assay for interleukin-13, interferon- $\gamma$, tumour necrosis factor- $\alpha$ and transforming growth factor- $\beta_{1}$ messenger ribonucleic acid

Total lung ribonucleic acid was extracted from frozen lungs using a commercial kit (ISOGEN, Nippon Gene Co., Toyama, Japan) according to the standard procedure [11]. A two-step reverse transcriptase polymerase chain reaction (RT-PCR) procedure was used according to the protocol of the TaqMan Gold RT-PCR Kit (Perkin-Elmer Applied Biosystems, Foster City, CA, USA). The primers and probes for rat IL-13, interferon (IFN)- $\gamma$, tumour necrosis factor (TNF)- $\alpha$ and transforming growth factor (TGF)- $\beta_{1}$ were defined using primer express software (Perkin-Elmer Applied Biosystems) (table 1). As a housekeeping gene, glyceraldehyde-3-phosphate dehydrogenase (GAPDH) mRNA was also amplified at the same time using TaqMan rodent GAPDH control

Table 1.-Primers and probes used for real-time quantitative reverse transcriptase polymerase chain reaction

\begin{tabular}{cc}
\hline IL-13 & 5'CCTGGAATCCCT \\
F-primer & GACCAACAT3' \\
R-primer & 5'CAGAGGCCATTC \\
Probe & AATATCCTCTG3' \\
5'TCCAGTTGCAATG \\
IFN- $\gamma$ CCATCCACAGGAC3' \\
F-primer & 5'ACCAGCTGTCACC \\
R-primer & AGAATCTAGC3' \\
& 5'TGGCACACTCTC \\
Probe & TACCCCAGAA3' \\
TNF- $\alpha$ & 5'TAAGGAAGCGGAAAA \\
F-primer & GGAGTCGGTGCTG3' \\
R-primer & 5'GCTCCCTCTCAT \\
Probe & CAGTTCCATG3' \\
TGF- $\beta_{1}$ & 5'TTGGTGGTTTGC \\
F-primer & TACGACGTG3' \\
R-primer & 5'CCCAGACCCTCACACT \\
Probe & CAGATCATCTTCT3' \\
& 5'GCTTCAGCTCCA \\
& CAGAGAAGAA3' \\
& 5'TCCAACCCAGG \\
TCCTTCCTAA3' & 5'TGCTGTGTACGGCAG \\
& CTGTACATTGAC3'
\end{tabular}

IL: interleukin; IFN: interferon; TNF: tumour necrosis factor; TGF: transforming growth factor; F: forward; R: reverse. reagent (Perkin-Elmer Applied Biosystems). Reverse transcription was performed at $48^{\circ} \mathrm{C}$ for $30 \mathrm{~min}$. Polymerase chain reaction (PCR) amplification and simultaneous quantification of a target sequence were then carried out using the PRISM 7700 Sequence Detector (Perkin-Elmer Applied Biosystems). PCR was performed for $10 \mathrm{~min}$ at $95^{\circ} \mathrm{C}$, followed by 40 cycles of amplification $\left(95^{\circ} \mathrm{C}\right.$ for $15 \mathrm{~s}$ and $60^{\circ} \mathrm{C}$ for $90 \mathrm{~s})$ for all samples in duplicate. Details of this method are described in previous reports $[12,13]$. The absolute number of transcript copies was normalized to an endogenous control, the GAPDH transcript. Results were expressed as a relative ratio for the mean level of the single-sham group.

\section{Statistical analysis}

Because the results were not normally distributed, the OA-challenged groups were compared with the control groups by the nonparametric Mann-Whitney U-test. A difference was considered to be significant when $\mathrm{p}<0.05$. All results in the text, tables and figures are presented as mean \pm SEM.

\section{Results}

Airway responsiveness to methacholine and ovalbumin

Airway responsiveness either to $\mathrm{Mch}$ or to $\mathrm{OA}$ was significantly increased in the single-OA group compared with the single-sham group (figs. 1 and 2). Both $\log \mathrm{PC} 150 \mathrm{Mch}$ and the $\%$ increase of $P$ ao gradually declined over time, when the rats received repeated saline challenges. However, repeated antigen challenges significantly attenuated the time-dependent decline in $\log \mathrm{PC} 150 \mathrm{Mch}$, so that both $1 \mathrm{M}-\mathrm{OA}$ and 3M-OA groups showed significantly higher nonspecific AHR than the control groups. By contrast, repeated antigen challenges significantly accelerated

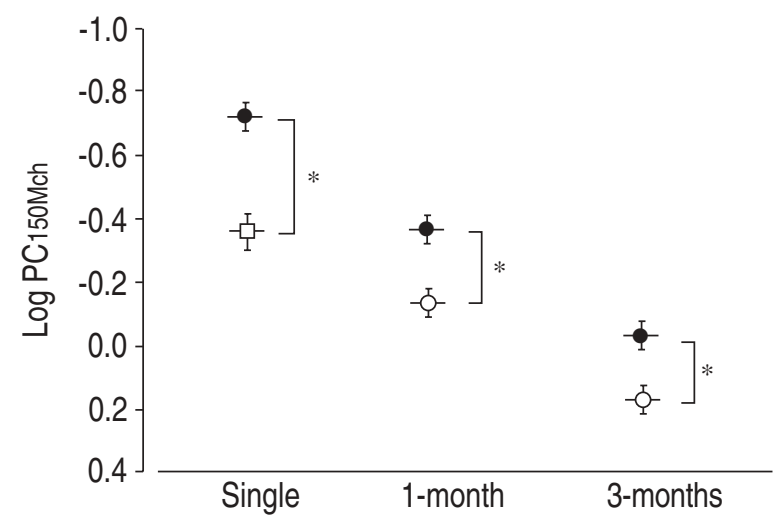

Fig. 1.-Airway responsiveness to methacholine (Mch) after single, 1 -month and 3-month antigen challenges in rats. PC150Mch: Mch concentration needed to induce a $50 \%$ increase from baseline airway opening pressure. $\square$ : unsensitized sham controls in the singly-challenged group; $\bigcirc$ : sensitized and saline-challenged controls; : sensitized and ovalbumin-challenged groups. Data are presented as mean \pm SEM. $*$ : $\mathrm{p}<0.05$. 


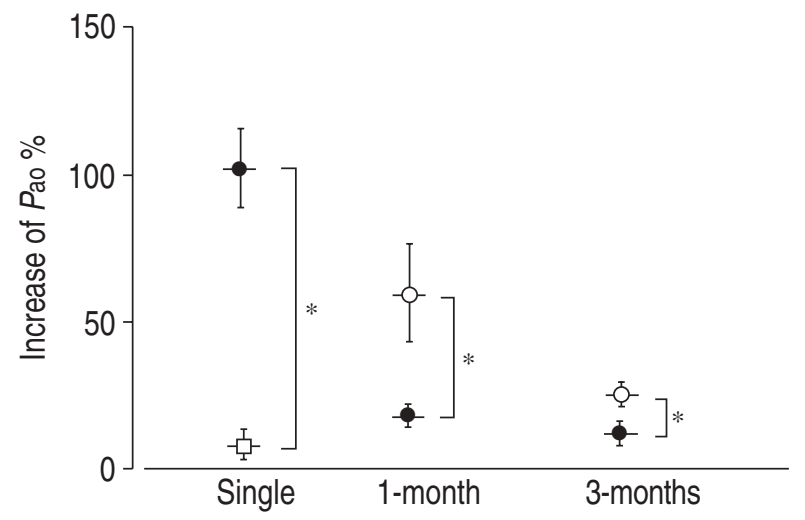

Fig. 2.-Airway responsiveness to ovalbumin (OA) after single, 1 -month and 3-month antigen challenges in rats. Data are expressed as percentages of increased airway opening pressure $(P$ ao $)$ (after/before OA inhalation for $10 \mathrm{~min}$ ). $\square$ : unsensitized sham controls in the singly-challenged group; $\bigcirc$ : sensitized and saline-challenged controls; $\bigcirc$ : sensitized and OA-challenged groups. Data are presented as mean \pm SEM. *: $\mathrm{p}<0.05$.

the time-dependent decline in the $\%$ increase of $P$ ao after antigen inhalation, so that both $1 \mathrm{M}-\mathrm{OA}$ and 3M-OA groups showed significantly lower antigenspecific AHR than the control groups.

\section{Morphometrical analysis}

A significant difference was noted between the $1 \mathrm{M}$ OA group and the $1 \mathrm{M}$-control group, only in the smooth muscle as well as the walls and epithelia of the small airways (fig. 3). Despite such positive effects of a 1-month OA challenge on airway morphology, there were no significant differences in any parameters of airway morphology between the 3M-OA group and the 3M-control group.

\section{Total and differential cell counts of lavaged cells}

In the single-OA group, there was a six-fold increase in the total cell number compared with the single-sham group (table 2). Differential cell counts revealed that alveolar macrophages and eosinophils were generally responsible for the increase in total cell number. After repeated antigen or saline challenges, however, there were no significant differences between the two groups in the number of eosinophils, because eosinophils disappeared whether the rats received repeated antigen challenges or not. A significant difference was noted between the two groups in the number of alveolar macrophages (i.e. both $1 \mathrm{M}-\mathrm{OA}$ and 3M-OA groups showed significantly higher numbers of alveolar macrophages than the control groups).

The numbers of CD4+ and CD8+ lymphocytes were also significantly increased in the single-OA and 1M-OA groups compared with the control groups. However, there were no significant differences in these cell counts between the repeatedly antigen-challenged rats and the control rats at 3 months (table 3 ). a)
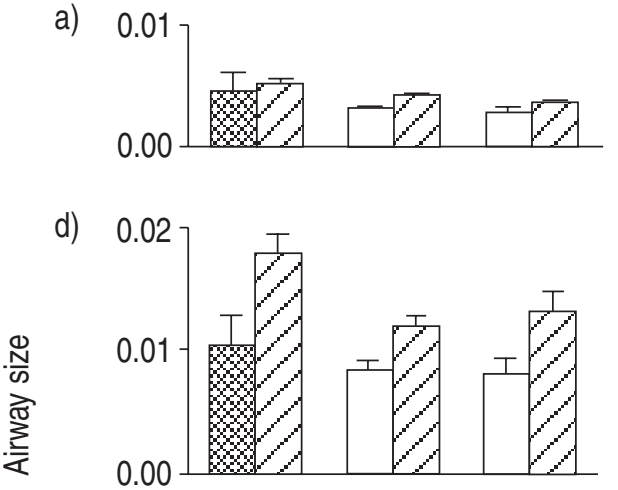

g) 0.02

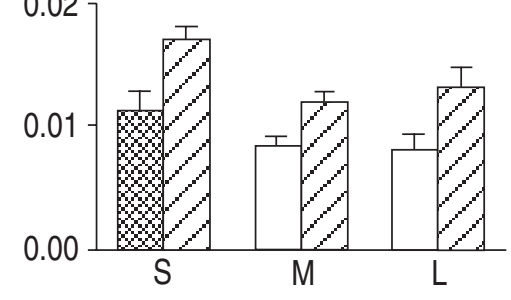

b)

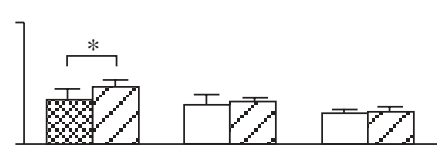

e)

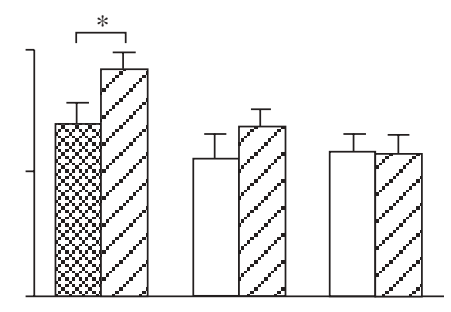

h)

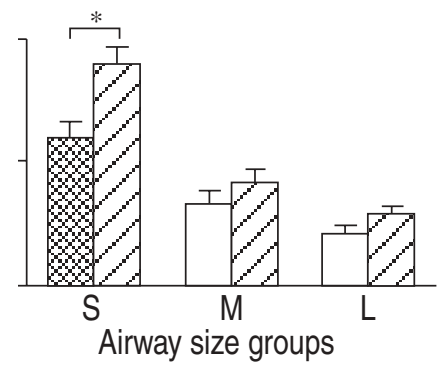

c)

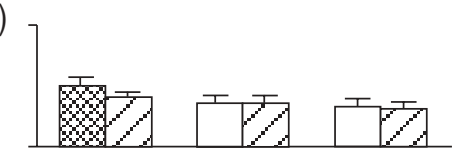

f)

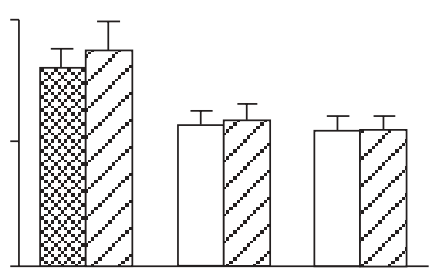

i)

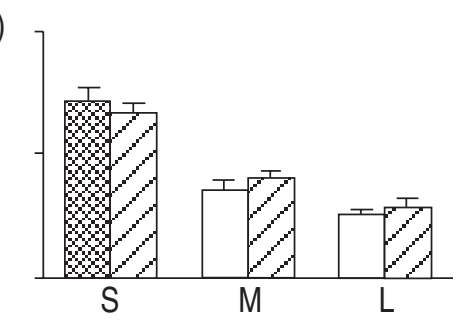

Fig. 3. - Morphometrical dimensions of the airways after single (a, d, g), 1-month (b, e, h) and 3-month (c, f, i) antigen challenges in rats. Y-axes show area collected by airway size (area divided by the length ${ }^{2}$ of the basement membrane): a-c) smooth muscle; $d-f$ ) wall; $g-i$ ) epithelium. S: small airways; M: middle-sized airways; L: large airways. sensitized and saline-challenged controls in repeatedly-challenged groups; $\mathbb{Z}$ : sensitized and ovalbumin-challenged groups. Data are presented as mean \pm SEM. *: $\mathrm{p}<0.05$. 
Table 2. - Total and differential cell counts in bronchoalveolar lavage fluid (BALF)

\begin{tabular}{|c|c|c|c|c|c|c|}
\hline Group & $\mathrm{n}$ & Total cells & МФ & Lymph & Eosinophils & Neutrophils \\
\hline Single-sham & 8 & $88 \pm 19$ & $78 \pm 17$ & $0.5 \pm 0.2$ & $9.6 \pm 5.7$ & $0.09 \pm 0.05$ \\
\hline Single-OA & 8 & $520 \pm 47 * * *$ & $332 \pm 32 * * *$ & $18.4 \pm 4.9 * * *$ & $169.5 \pm 33.1 * * *$ & $0.00 \pm 0.00$ \\
\hline $1 \mathrm{M}$-cont & 8 & $143 \pm 12$ & $140 \pm 12$ & $1.4 \pm 0.4$ & $1.6 \pm 0.3$ & $0.48 \pm 0.16$ \\
\hline $1 \mathrm{M}-\mathrm{OA}$ & 8 & $353 \pm 43^{\#}$ & $344 \pm 42^{\#}$ & $6.4 \pm 1.2^{\#}$ & $2.1 \pm 0.5$ & $0.25 \pm 0.18$ \\
\hline $3 \mathrm{M}$-cont & 8 & $183 \pm 16$ & $176 \pm 15$ & $3.8 \pm 0.7$ & $2.4 \pm 0.7$ & $1.65 \pm 0.54$ \\
\hline $3 \mathrm{M}-\mathrm{OA}$ & 8 & $309 \pm 25^{* *}$ & $298 \pm 26^{* *}$ & $5.7 \pm 1.2$ & $3.9 \pm 1.1$ & $0.20 \pm 0.20 *$ \\
\hline
\end{tabular}

Data are presented as mean \pm SEM and number of cells $\left(\times 10^{4}\right)$ in $1 \mathrm{~mL}$ of BALF; OA: ovalbumin; $1 \mathrm{M}$ : 1 month; $3 \mathrm{M}$ : 3 months; cont: control. *: $\mathrm{p}<0.05$ compared with $3 \mathrm{M}$-cont; **: $\mathrm{p}<0.01$; $^{*}: \mathrm{p}<0.01$ compared with $1 \mathrm{M}$-cont; ***: $\mathrm{p}<0.001$ compared with single-sham.

Expression of messenger ribonucleic acid for interleukin-13, interferon- $\gamma$, tumour necrosis factor- $\alpha$ and transforming growth factor- $\beta_{1}$

Only the level of TGF- $\beta_{1}$ mRNA among the four cytokines examined was significantly upregulated in the $3 \mathrm{M}-\mathrm{OA}$ compared with the $3 \mathrm{M}$-control group (fig. 4).

\section{Discussion}

In the present study, the authors demonstrated dissociation between nonspecific AHR and antigenspecific AHR after repeated antigen challenges in $\mathrm{BN}$ rats. The two types of AHR were modulated in opposite directions by repeated antigen challenges in rats sensitized with ovalbumin. The time-dependent decline in antigen-specific AHR was significantly accelerated by repeated antigen challenges, indicating the occurrence of desensitization. In contrast, the natural time-dependent decline in nonspecific AHR after one-time sensitization was significantly attenuated by repeated antigen challenges. The number of alveolar macrophages in BALF and the expression of TGF- $\beta_{1}$ mRNA were the only factors examined in the present study that might explain the dissociation of the two AHRs with repeated antigen challenges.

A number of previous studies demonstrated that multiple antigen challenges generated a marked increase in nonspecific AHR in BN rats [6-8]. On the other hand, several studies examining the immunological aspects of multiple antigen challenge demonstrated in the same strain of rats that such

Table 3. - Number of CD4+ and CD8+ lymphocytes

\begin{tabular}{lccc}
\hline Group & $\mathrm{n}$ & $\mathrm{CD} 4$ & $\mathrm{CD} 8$ \\
& & & \\
\hline Single-sham & 8 & $1.9 \pm 0.9$ & $0.3 \pm 0.1$ \\
Single-OA & 8 & $124.5 \pm 30.7^{* * *}$ & $17.7 \pm 7.9^{* * *}$ \\
1M-cont & 8 & $7.7 \pm 3.0$ & $1.0 \pm 0.3$ \\
1M-OA & 8 & $28.1 \pm 5.2^{\#}$ & $10.1 \pm 2.0^{\#}$ \\
3M-cont & 8 & $23.6 \pm 4.5$ & $5.0 \pm 0.9$ \\
3M-OA & 8 & $30.7 \pm 7.1$ & $7.9 \pm 2.0$ \\
\hline
\end{tabular}

Data are presented as mean \pm SEM and number of CD4+ and $\mathrm{CD} 8+\left(\times 10^{3}\right)$ in $1 \mathrm{~mL}$ of bronchoalveolar lavage fluid. OA: ovalbumin; $1 \mathrm{M}$ : 1 month; $3 \mathrm{M}: 3$ months; cont: control. ***: $\mathrm{p}<0.001$ compared with single-sham; ${ }^{\#}: \mathrm{p}<0.001$ compared with $1 \mathrm{M}$-cont. challenges might induce IgE isotype-specific tolerance $[9,10]$. Tolerance to a specific antigen should result in the suppression of eosinophilic inflammation of the airways, and thus, also the suppression of antigenspecific AHR. Accordingly, the authors attempted to prove the dissociation of nonspecific AHR from antigen-specific AHR in the same group of rats. To the authors' best knowledge, this is the first study providing evidence that such dissociation really occurs in the same animals.

An attempt was made to examine the mechanism underlying the dissociation. To study the profile of inflammatory cells in BALF would explain which cell type was involved in the enhancement of nonspecific AHR and/or the suppression of antigen-specific AHR with repeated antigen challenges. In the present study, alveolar macrophages were the only cell type that was significantly increased in rats receiving repeated antigen challenges, compared with the control rats receiving only saline instead. It is well known that macrophages have the potential to release proinflammatory mediators, including leukotriene $\mathrm{B}_{4}$, platelet activating factor and nitric oxide, which have an important role on bronchial hyperresponsiveness $[14,15]$. Thus, it can be speculated that airway inflammation induced by macrophages may play a role in the enhancement of nonspecific AHR in the present study's model rats receiving multiple antigen challenges. This speculation is supported by a human study that demonstrated a correlation of nonspecific AHR with the number of alveolar macrophages in BALF in asthmatic children [16]. On the other hand, some evidence is available suggesting that macrophages are also involved in inducing immunological tolerance [17, 18]. Accordingly, an increase in the number of alveolar macrophages may also have contributed to the suppression of antigen-specific AHR in the present experiment. By contrast, eosinophilic inflammation of the airways, elicited after the single antigen challenge, completely disappeared after repeated antigen challenges. This indicated that eosinophils were not responsible for the sustained increase in nonspecific AHR in the rats receiving repeated antigen challenges.

Another mechanism that may account for enhanced nonspecific AHR with repeated antigen challenges is morphological changes of the airways. In asthma, thickening of airway smooth muscles may lead to narrowing of the airways [19], and thus contribute to 

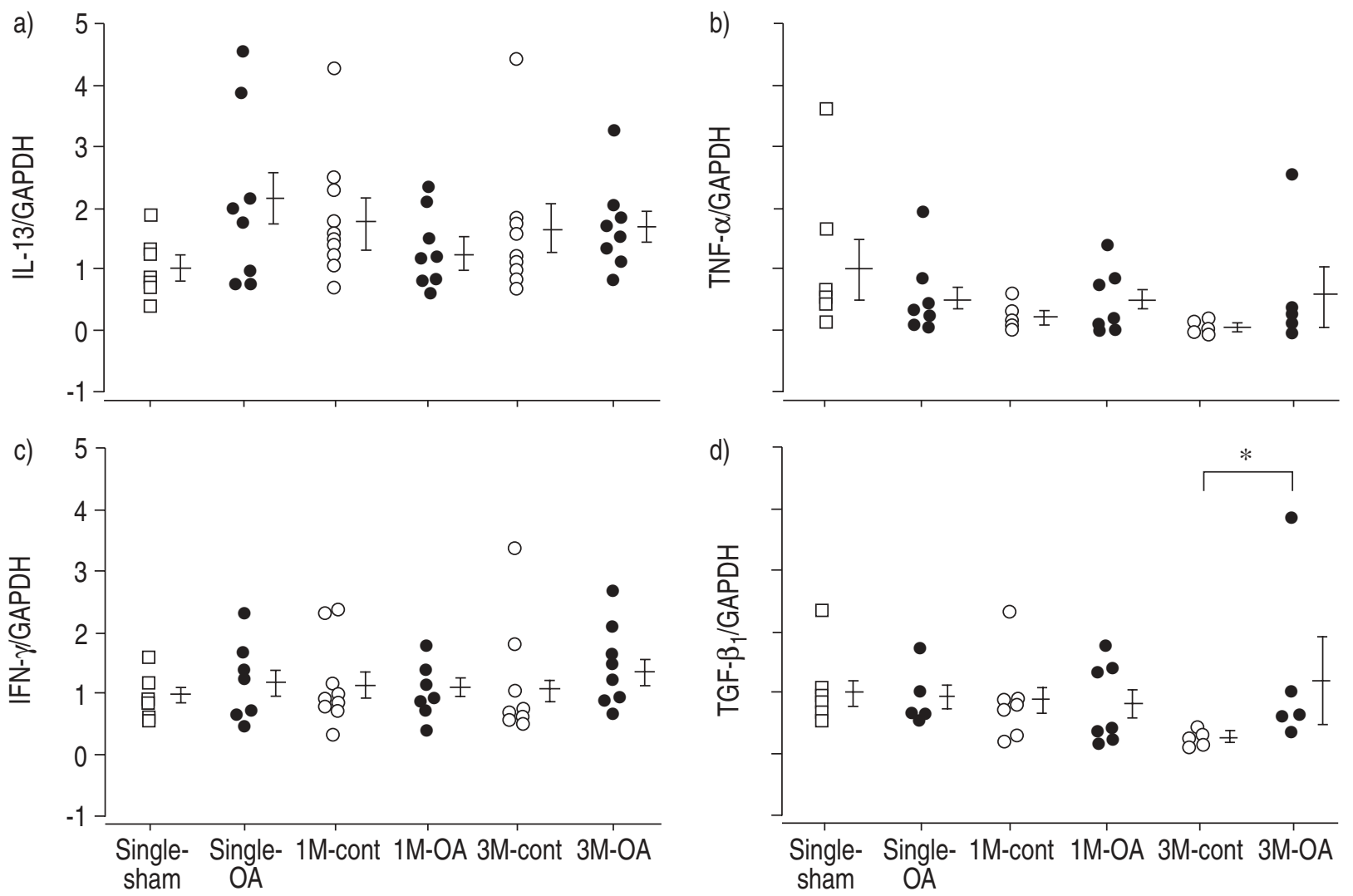

Fig. 4. - Expression of messenger ribonucleic acid for a) interleukin (IL)-13, b) tumour necrosis factor (TNF)- $\alpha$, c) interferon (IFN)- $\gamma$, and d) transforming growth factor (TGF)- $\beta_{1}$ after single and repeated antigen challenges in rats. Y-axes show relative ratios to the singlesham group. $\square$ : unsensitized sham controls in the singly-challenged group; $\bigcirc$ : sensitized and saline-challenged controls in repeatedlychallenged groups; 0 : sensitized and ovalbumin (OA)-challenged rats. GAPDH: glyceraldehyde-3-phosphate dehydrogenase; cont: control; 1M: 1 month; 3M: 3 months. Data are presented as mean \pm SEM. *: $\mathrm{p}<0.05$.

nonspecific AHR. This mechanism was postulated to be true in previous experiments, which used $\mathrm{BN}$ rats treated in a way similar to the present experiment [4, 5]. Contrary to the present authors' expectation, however, the morphological changes of the airways observed after 1 month of antigen challenges disappeared after 3-month repeated antigen challenges; and there were no significant differences in the morphology of the airways between the repeatedly antigen-challenged rats and the control rats. The reason why it was not possible to develop airway remodelling may be explained by differences in the experimental protocol. The present authors used a larger amount of inhaled antigen, and/or gave it to the animals more frequently, for a relatively longer period than in previously published reports. However, the possibility of any contribution of airway remodelling to the enhancement of nonspecific AHR cannot be completely denied, because there may have been morphological changes in the airways smaller than those examined in the present study.

Previous studies suggested that cytokines such as IL-13, IFN- $\gamma$ and TNF- $\alpha$ are involved in the development of nonspecific AHR in murine models of atopic asthma [20-23]. TGF- $\beta_{1}$ is a growth factor that is believed to play an important role in the development of airway remodelling in asthma, thus contributing to the development of nonspecific AHR [24]. TGF- $\beta_{1}$, on the other hand, was reported to suppress nonspecific AHR in a murine model of atopic asthma [25], and it may also be responsible for the development of immunological tolerance [25, 26]. Accordingly, TGF- $\beta_{1}$ is a potential candidoate accounting for the dissociation of nonspecific AHR from antigen-specific AHR. Indeed, the present authors found that only the level of TGF- $\beta_{1}$ mRNA was significantly upregulated among the four cytokines examined for the whole lung tissue in rats receiving repeated antigen challenges compared to controls. Although it remains to be clarified whether upregulation of TGF- $\beta_{1}$ mRNA is really linked to the enhancement of nonspecific AHR with repeated antigen challenges, this cytokine may prove to play a key role in the dissociation of nonspecific AHR from antigen-specific AHR.

As discussed previously, it appears that a number of factors are involved in the development of nonspecific AHR. It is highly likely that the nonspecific AHR in the inflammatory airways depends on the balance of the protective and worsening factors. Thus, the authors feel that the present study actually reflects such complexity of the development of nonspecific AHR.

Antigen-specific immunotherapy is a strategy for 
the treatment of allergic asthma in children. However, the efficacy of immunotherapy has not been universally accepted. The National Institutes of Health (NIH) International Consensus Report on Diagnosis and Management and the World Health Organization (WHO)/NIH Global Initiative on Asthma Management recommend that allergen immunotherapy should only be considered when asthma is poorly controlled with drugs, and where allergen avoidance is impossible [27, 28]. There has been at least one report, Murray et al. [29], which demonstrated the enhancement of nonspecific AHR by more than two-fold after mite immunotherapy in patients with atopic asthma. Although it may be premature to apply the results of the present study directly to humans, the animal model presented in this report may help to clarify why allergen immunotherapy is not always as effective as expected.

In conclusion, the authors have demonstrated a dissociation of airway hyperresponsiveness to methacholine from antigen-specific airway hyperresponsiveness in brown Norway rats that received repeated antigen challenges after ovalbumin sensitization. Sustained airway inflammation with macrophages and/or upregulation of transforming growth factor$\beta_{1}$ messenger ribonucleic acid in the lung tissue may be responsible for this dissociation.

Acknowledgements. The authors would like to thank Y. Kawakami (Konan Hospital, Sapporo, Japan) for his constructive comments and E. Yamaguchi (The First Dept of Medicine, School of Medicine, Hokkaido University, Sapporo, Japan) for his valuable advice regarding the flow cytometry technique.

\section{References}

1. Waserman S, Olivenstein R, Renzi P, Xu L, Martin JG. The relationship between late asthmatic responses and antigen-specific immunoglobulin. J Allergy Clin Immunol 1992; 90: 661-669.

2. Haczku A, Macary P, Haddad EB, et al. Expression of Th-2 cytokines interleukin- 4 and -5 and of Th- 1 cytokine interferon- $\gamma$ in ovalbumin-exposed sensitized Brown-Norway rats. Immunology 1996; 88: 247-251.

3. Elwood W, Barnes PJ, Chung KF. Airway hyperresponsiveness is associated with inflammatory cell infiltration in allergic Brown-Norway rats. Int Arch Allergy Immunol 1992; 99: 91-97.

4. Sapienza S, Du T, Eidelman DH, Wang NS, Martin JG. Structural changes in the airways of sensitized Brown Norway rats after antigen challenge. Am Rev Respir Dis 1991; 144: 423-427.

5. Cui ZH, Skoogh BE, Pulleritis T, Lotvall J. Bronchial hyperresponsiveness and airway wall remodelling induced by exposure to allergen for 9 weeks. Allergy 1999; 54: 1074-1082.

6. Bellofiore S, Martin JG. Antigen challenge of sensitized rats increases airway responsiveness to methacholine. J Appl Physiol 1988; 65: 1642-1646.

7. Haczku A, Chung KF, Sun J, Barnes PJ, Kay AB, Moqbel R. Airway hyperresponsiveness, elevation of serum-specific $\mathrm{IgE}$ and activation of $\mathrm{T}$ cells following allergen exposure in sensitized Brown-Norway rats. Immunology 1995; 85: 598-603.

8. Haczku A, Moqbel R, Elwood W, et al. Effects of prolonged repeated exposure to ovalbumin in sensitized Brown Norway rats. Am J Respir Crit Care Med 1994; 150: 23-27.

9. Sedgwick JD, Holt PG. Induction of IgE-isotype specific tolerance by passive antigen stimulation of the respiratory mucosa. Immunology 1983; 50: 625-630.

10. Sedgwick JD, Holt PG. Suppression of IgE responses in inbred rats by repeated respiratory tract exposure to antigen: responder phenotype influences isotype specificity of induced tolerance. Eur J Immunol 1984; 14: 893-897.

11. Varela LM, Ip MM. Tumor necrosis factor-alpha: a multifunctional regulator of mammary gland development. Endocrinology 1996; 137: 4915-4925.

12. Heid CA, Stevens J, Livak KJ, Williams PM. Real time quantitative PCR. Genome Res 1996; 6: 986-994.

13. Luthra R, McBride JA, Cabanillas F, Sarris A. Novel $5^{\prime}$ exonuclease-based real-time PCR assay for the detection of $\mathrm{t}(14 ; 18)(\mathrm{q} 32 ; \mathrm{q} 21)$ in patients with follicular lymphoma. Am J Pathol 1998; 153: 63-68.

14. Cuss FM, Dixon CMS, Barnes PJ. Effects of inhaled platelet activating factor on pulmonary function and bronchial responsiveness in man. Lancet 1986; 2: 189-192.

15. Andrew JC, Geoffrey AS, Andrew SM. Alveolar macrophages bind and phagocytose allergen-containing pollen starch granules via C-type lectin and integrin receptors: implication for airway inflammatory disease. J Immunol 2000; 164: 3878-3886.

16. Ferguson AC, Wong FWM. Bronchial hyperresponsiveness in asthmatic children. Correlation with macrophages and eosinophils in broncholavage fluid. Chest 1989; 96: 988-991.

17. Attwood JT, Munn DH. Macrophage suppression of $\mathrm{T}$ cell activation: a potential mechanism of peripheral tolerance. Int Rev Immunol 1999; 18: 515-525.

18. Thepen T, Van RN, Kraal G. Alveolar macrophage elimination in vivo is associated with an increase in pulmonary immune response in mice. $J$ Exp Med 1989; 170: 499-509.

19. James AL, Paré PD, Hogg JC. The mechanics of airway narrowing in asthma. Am Rev Respir Dis 1989; 139: 242-246.

20. Wills-Karp M, Luyimbazi J, Xu X, et al. Interleukin13: central mediator of allergic asthma. Science 1998; 282: 2258-2261

21. Grünig G, Warnock M, Wakil AE, et al. Requirement for IL-13 independently of IL-4 in experimental asthma. Science 1998; 282: 2261-2263.

22. Hessel EM, Antoon J M, Van O, et al. Development of airway hyperresponsiveness is dependent on interferon- $\gamma$ and independent of eosinophil infiltration. Am J Respir Cell Mol Biol 1997; 16: 325-334.

23. Parris JR, Cobban HJ, Littlejohn AF, MacEwan DJ, Nixon GF. Tumor necrosis factor-alpha activates a calcium sensitization pathway in guinea-pig bronchial smooth muscle. J Physiol 1999; 518: 561-569.

24. Minshall EM, Leung DY, Martin RJ, et al. Eosinophilassociated TGF-beta1 mRNA expression and airways fibrosis in bronchial asthma. Am J Respir Cell Mol Biol 1997; 17: 326-333.

25. Hansen G, McIntire JJ, Yeung VP, et al. CD4(+) T helper cells engineered to produce latent TGF-beta1 reverse allergen-induced airway hyperreactivity and inflammation. J Clin Invest 2000; 105: 61-70. 
26. Meade R, Askenase PW, Geba GP, Neddermann K, Jacoby RO, Pasternak RD. Transforming growth factor- $\beta_{1}$ inhibits murine immediate and delayed type hypersensitivity. J Immunol 1992; 149: 521-528.

27. Guidelines for the diagnosis and management of asthma. National Heart, Lung and Blood Institute. National Asthma Education Program. Expert Panel Report. J Allergy Clin Immunol 1991; 88: 425534.
28. Global strategy for asthma management and prevention. National Heart, Lung and Blood Institute/World Health Organization: Global Initiative for Asthma. 1995. Bethesda, MD, National Institutes of Health, Publication No. 95-3659.

29. Murray AB, Ferguson AC, Morrison BJ. Non-allergic bronchial hyperreactivity in asthmatic children decreases with age and increases with mite immunotherapy. Ann Allergy 1985; 54: 541-544. 